\title{
THIN FILM EVOLUTION OVER A THIN POROUS LAYER: MODELING A TEAR FILM ON A CONTACT LENS*
}

\author{
KUMNIT NONG ${ }^{\dagger}$ AND DANIEL M. ANDERSON ${ }^{\dagger}$
}

\begin{abstract}
We examine a mathematical model describing the behavior of the precontact lens tear film of a human eye. Our work examines the effect of contact lens thickness and lens permeability on the film dynamics. Also investigated are gravitational effects and the effects of different slip models at the fluid-lens interface. A mathematical model for the evolution of the tear film is derived using a lubrication approximation applied to the hydrodynamic equations of motion in the fluid film and the porous layer. The model is a nonlinear fourth-order partial differential equation subject to boundary conditions and an initial condition for post-blink film evolution. The evolution equation is solved numerically, and the effects of various parameters on the rupture of the thin film are studied. We find that increasing the lens thickness, permeability, and slip all contribute to an increase in the film thinning rate, although for parameter values typical for contact lens wear, these modifications are minor. Gravity plays a role similar to that for tear films in the absence of a contact lens. The presence of the contact lens does, however, fundamentally change the nature of the rupture dynamics as the inclusion of the porous lens leads to rupture in finite time rather than infinite time.
\end{abstract}

Key words. thin films, tear film, contact lens, porous layer, fluid porous slip, interface slip

AMS subject classifications. 76A20, 76S05, 92C30, 92C50

DOI. $10.1137 / 090749748$

1. Introduction. The human tear film is a complex fluid system whose presence is required for both proper vision as well as the overall health of the eye. A common view of the human tear film characterizes it as a medium composed of three distinct layers over the corneal surface: an innermost mucus layer on the cornea, an intermediate aqueous layer, and an outermost lipid or fatty layer acting as a final barrier to the outside environment (e.g., Sharma, Khanna, and Reiter [1], Zhang, Matar, and Craster [2], and Braun and Fitt [3]). More recent views (Gipson [4], Bron et al. [5], and Cher [6]) suggest a somewhat more complex system. In particular, the current view replaces the mucus and aqueous layers with a mucoaqueous layer in which mucins secreted from goblet cells are distributed throughout the bulk of the tear film and epithelial mucins form a complex barrier at the corneal surface. Measurements of the overall thickness of a human tear film range from a few microns to as many as 40 (see the review by Bron et al. [5]). The corneal surface itself has 100-nm-scale microbumps and rod-like mucins of length 200-500 $\mathrm{nm}$ that extend into the tear film [4]. These mucins have multiple functions ranging from cleanup and removal of debris from the tear film, stabilization of the tear film, and, with particular attention to the membrane-associated mucins, maintaining wettability at the corneal surface. The lipid layer, whose thickness has been estimated to range between 13-100 nm, serves to further stabilize the film and to slow evaporative mass loss (Bron et al. [5]).

Dry eye syndrome is a common disorder of the human tear film that results from decreased tear production, excessive tear evaporation, and/or an abnormality in the

*Received by the editors February 17, 2009; accepted for publication (in revised form) June 22, 2010; published electronically August 19, 2010. This work was supported by the U.S. National Science Foundation, Computational Science Training for Undergraduates in the Mathematical Sciences, DMS-0639300.

http://www.siam.org/journals/siap/70-7/74974.html

${ }^{\dagger}$ Department of Mathematical Sciences, George Mason University, Fairfax, VA 22030 (knong@ gmu.edu, danders1@gmu.edu). The second author's research was supported by the Applied Mathematics Program, U.S. National Science Foundation grant DMS-0709095.

2771 
production of mucus and lipids (Lemp et al. [7]). Without a sufficient tear film, eye irritation may occur and can lead to more severe damage of the corneal surface. The understanding of diseases such as dry eye syndrome has led to a growing interest in the applied mathematics and fluid dynamics community in developing models that allow for quantitative prediction of tear film thinning and rupture addressing issues such as aqueous layer stability (Sharma and Ruckenstein [8]), the dynamics of tear deposition and thinning due to lid motion (Wong, Fatt, and Radke [9]), mucus layer stability (Sharma, Khanna, and Reiter [1]), non-Newtonian rheology of the tear film (Zhang, Matar, and Craster [2]), evaporation and gravitational drainage (Braun and Fitt [3]), evaporation and corneal surface wetting (Winter, Anderson, and Braun [10]), dynamics during blink cycles (Heryudono et al. [11], Braun and King-Smith [12]), reflex tearing (Maki et al. [13]) and two-dimensional eye-shaped domains (Maki et al. $[14,15])$.

For wearers of contact lenses, the presence of an ample post-lens tear film (between the cornea and the contact lens) as well as an ample pre-lens tear film (between the contact lens and the outside environment) are critical to maintaining the overall health of the eye and enabling the proper corrective function of the contact lens. Certainly the introduction of a contact lens further complicates the basic geometry of the tear film and introduces the possibility of multiple layers (mucoaqueous and lipid) in the pre-lens and post-lens films. Recent measurements of pre- and post-lens tear films suggest a value near $2.3 \mu \mathrm{m}$ for each (Nichols and King-Smith [16]) and, in general, different thinning rates for pre- and post-lens films (Nichols and King-Smith [17] and Nichols, Mitchell, and King-Smith [18]).

Contact lenses were first manufactured and worn in the late 1800s; these designs ranged from "blown glass shells" and "lenses cut from crystal" to ground scleral lenses and were developed with the correction of various optical conditions in mind [19]. In addition to requirements involving the optical corrective function, modern contact lens material (e.g., hydrogel or gas-permeable soft contact lenses), design, and manufacture must also address issues such as lens-induced hypoxia caused by reduced transport of oxygen through the contact lens to the cornea, especially for long-term and continuous-wear lenses [20, 21, 22].

The motion of the contact lens during a blink, which influences the overall oxygen transport to the cornea, relies on sufficient pre- and post-lens tear films for lubrication and the avoidance of abrasion of the cornea (Raad and Sabau [23]). The identification of mechanisms that cause settling of the contact lens during a blink is important for the understanding of how a stable post-lens tear film is maintained. Monticelli, Chauhan, and Radke [24] have assessed the influence of the hydraulic permeability of soft contact lenses on settling rates of a contact lens during a blink. They identify the settling rate of a rigid porous disk relative to that of an impermeable one. For typical values of the permeability for three different soft contact lenses (all approximately $10^{-8} \mu \mathrm{m}^{2}$ ), their results indicate a negligible influence of the permeability on the settling rate during a blink. The influence of deformation of the contact lens, modeled as a thin elastic shell, during multiple blink cycles and the question of a balance, or lack thereof, between the loss/gain of fluid in the post-lens tear film over a blink cycle has also been assessed (Chauhan and Radke [25]).

Dry eye syndrome may also occur for wearers of contact lenses and, in some cases, may prevent individuals from wearing contact lenses. Fornasiero, Prausnitz, and Radke [26] used a diffusion-based model to study depletion of the post-lens tear film due to evaporation of the pre-lens tear film and transport through the lens. The mechanism suggested for post-lens depletion was that once rupture of the pre-lens film 
occurred, evaporation of water from the lens begins and causes a flux of water away from the post-lens film. The net effect of evaporation from the lens is a reduction of the lubricating post-lens film, leading to a number of undesirable conditions ranging from discomfort to lens adhesion. Their study involved examining water transport driven by pre-lens evaporation through low water content (38 wt. percent) as well as high water content (70 wt. percent) polymer-based soft contact lenses. Their study indicated that either of these lenses could dehydrate faster, depending on external conditions such as humidity and wind speed. Other issues related to contact lens wear include increased evaporation and thinning rates of pre-lens tear films and wettability of the contact lens (Thai, Tomlinson, and Doane [27], Mathers [28]), the connection between a dry or partially dry contact lens and incomplete blinking (McMonnies [29]), as well as the understanding of dryness symptoms in wearers and nonwearers of contact lenses (Chalmers and Begley [30]).

In the present study, our objective is to develop a mathematical model for a prelens tear film in order to address how the dynamics and rupture of this fluid film are influenced by the properties of the contact lens. Our model describes a thin liquid film (the pre-lens tear film) on a permeable contact lens modeled as a rigid porous layer of constant, finite thickness. In contrast to previous studies related to the thinning of the post-lens film, here our focus is on the possible influence of the presence of the contact lens on the dynamics and rupture of the pre-lens film. Consequently, we shall assume that (i) the lower boundary of the porous layer (lens) is impermeable and stationary so that the post-lens film is decoupled from the model and (ii) evaporation of the prelens film does not occur; only rupture due to capillarity, gravitational drainage, and the influence of the contact lens properties such as permeability, thickness, and slip are considered. Additionally, our focus will be on the dynamics of the film between blinks, that is, once the eyelid has opened and before the next blink. Our hope is that this mathematical model will provide (i) a setting in which the influence of various contact lens properties on the dynamics of the pre-lens film can be assessed and (ii) a basis upon which additional effects such as evaporation and the coupling of the pre-lens film dynamics to that in the post-lens film can be developed.

As such, our mathematical model bears some similarity to previous work on the dynamics of fluids on porous layers in other contexts that include contact line spreading (Davis and Hocking [31, 32]), dewetting (Devauchelle, Josser, and Zaleski [33]), and gravity current flows (Acton, Huppert, and Worster [34]), as well as thin film linear and nonlinear stability (Pascal [35], Sadiq and Usha [36]). In our work we shall investigate two basic models of slip on porous surfaces: the classical Beavers and Joseph slip condition [37, 38, 39, 40] used also in the contact lens model of Raad and Sabau [23], as well as a related one investigated more recently by Le Bars and Worster [41]. We address further details of this after the derivation of our basic model equations in the sections that follow.

The paper is organized as follows. The basic derivation of the mathematical model and all applied theories (e.g., lubrication theory, nondimensionalization) are given in section 2. The numerical results, in section 3, display the various effects of thickness of the porous layer, permeability, different types of slip at the fluid-porous interface, gravity, and film orientation. Section 4 includes a summary and discussion of the results. Finally, we include appendices in which further details of the analysis are given.

2. Formulation. Our model of the pre-lens film and contact lens is given by a layer of fluid above a fluid saturated porous medium as shown in Figure 1. The interface between the fluid layer and the porous layer is assumed to be planar and 


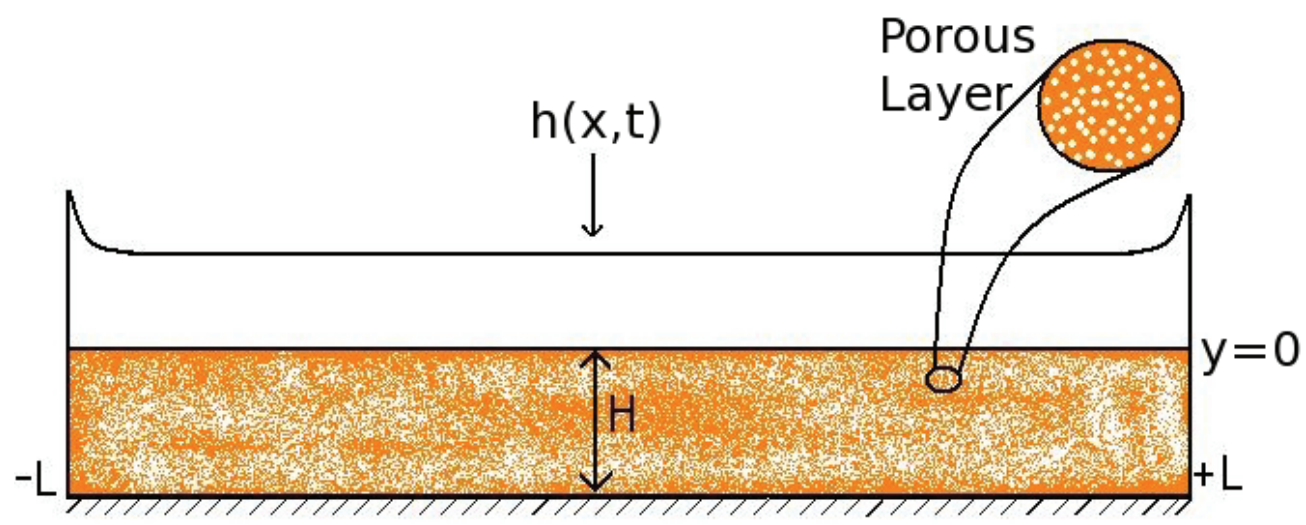

Corneal Surface

FIG. 1. Tear film on our porous contact lens model.

located at $y=0$. The surface of the fluid layer is located at $y=h(x, t)$. The lower boundary of the porous layer is assumed to be planar, impermeable, and located at $y=-H$, where $H>0$. We shall interpret $H$ as a typical thickness of a contact lens. The assumption that the contact lens is flat is in keeping with the common assumption in tear film models to neglect curvature of the corneal surface. Recent work by King-Smith et al. [42] has, in fact, calculated the influence of nonuniform curvature of the corneal surface and has shown its effect on thinning due to tangential flow in the tear film to be minor.

The derivation given below follows a standard lubrication theory modified to account for the presence of an underlying thin porous layer. This analysis is similar to recently published work by Sadiq and Usha [36]; however, some of the scaling assumptions, as well as the eventual evolution equation in their work, differ from ours. These differences include, for example, their assumption of an infinitely deep porous layer, as well as their approximation that the vertical component of the velocity at the fluid-porous boundary is negligible. Additionally, we derive evolution equations for two different slip models at the fluid-porous interface, as well as for a tangentially immobile free surface condition (see below and Appendices A and B).

The fluid is assumed to be Newtonian and incompressible with constant density $\rho$ and dynamic viscosity $\mu$. The governing equations in the liquid region $0<y<h(x, t)$ are given by

$$
\begin{aligned}
\nabla \cdot \boldsymbol{u} & =0 \\
\rho\left(\frac{\partial \boldsymbol{u}}{\partial t}+\boldsymbol{u} \cdot \nabla \boldsymbol{u}\right) & =-\nabla p+\mu \nabla^{2} \boldsymbol{u}-\rho g \cos \theta \hat{\mathbf{k}}+\rho g \sin \theta \hat{\mathbf{i}}
\end{aligned}
$$

where $\boldsymbol{u}=(u, v)$ is the two-dimensional velocity vector, $p$ is the fluid pressure, $g$ is gravitational acceleration, $\theta$ is the angle that the liquid-porous layer interface makes with the horizontal $(\theta=0$ corresponds to gravity pointing in the negative $y$ direction, while $\theta=\pi / 2$ corresponds to gravity pointing in the positive $x$ direction), and $\hat{\mathbf{k}}$ and $\hat{\mathrm{i}}$ are unit vectors in the $y$ and $x$ directions, respectively.

The porous medium is assumed to have constant permeability $k$ and uniform porosity, $1-\phi$, where $\phi$ is the solid volume fraction. The governing equations in the 


\section{Standard}



\section{Beavers- Joseph Le Bars \& Worster}

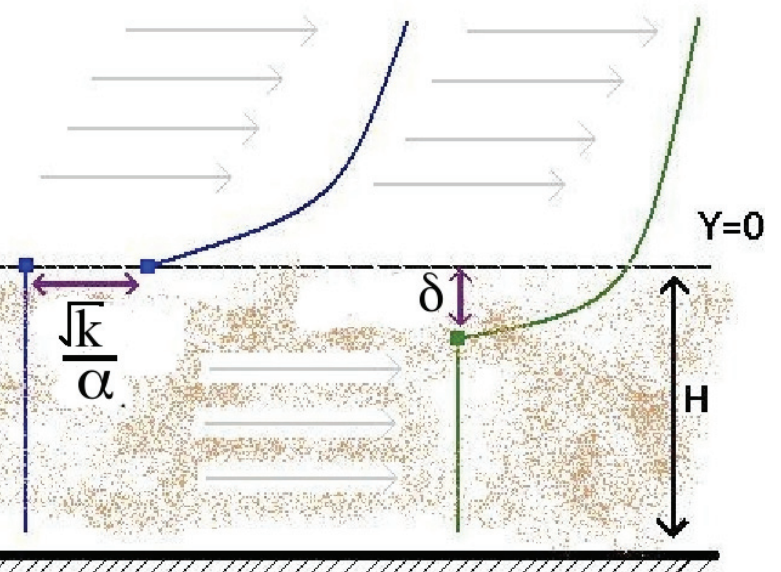

FIG. 2. Velocity profiles for various fluid-porous interface slip conditions (see also Le Bars and Worster [41, Figure 1]).

porous region $-H<y<0$ are given by

$$
\begin{aligned}
\nabla \cdot \boldsymbol{U} & =0, \\
\boldsymbol{U} & =-\frac{k}{\mu}(\nabla p+\rho g \cos \theta \hat{\mathbf{k}}-\rho g \sin \theta \hat{\mathbf{i}}),
\end{aligned}
$$

where $\boldsymbol{U}=(1-\phi) \boldsymbol{u}=(U, V)$ is the volume flow rate in the porous medium.

The above governing equations are subject to boundary conditions. At the impermeable boundary in the porous medium $y=-H$, we impose

$$
V=0 .
$$

At the liquid-porous boundary $y=0$, we impose the continuity conditions

$$
\begin{aligned}
v(x, y & \left.=0^{+}, t\right)=V\left(x, y=0^{-}, t\right), \\
\frac{\partial u}{\partial y}(x, y & \left.=0^{+}, t\right)=\frac{\alpha}{\sqrt{k}}\left(u\left(x, y=0^{+}, t\right)-U\left(x, y=0^{-}, t\right)\right), \\
p(x, y & \left.=0^{+}, t\right)=p\left(x, y=0^{-}, t\right) .
\end{aligned}
$$

The first of these represents a condition of mass conservation at this boundary. The third condition requires continuity of pressure. The second is the Beavers-Joseph condition [37] where $\alpha$ is a dimensionless constant; this slip condition was also used in the contact lens model of Raad and Sabau [23]. A recent alternative interfacial slip condition for a porous-fluid boundary that we also consider here was developed and examined for some simple flows by Le Bars-Worster [41]. Relevant details associated with the Le Bars and Worster boundary condition are included in Appendix A. A sketch of the physical interpretation of these slip boundary conditions, following that of Le Bars and Worster, is shown in Figure 2. At the liquid-vapor interface $y=h(x, t)$, we require that

$$
\begin{aligned}
\boldsymbol{u} \cdot \hat{\mathbf{n}} & =\boldsymbol{u}_{I} \cdot \hat{\mathbf{n}}, \\
\hat{\mathbf{n}} \cdot \mathbf{T} \cdot \hat{\mathbf{n}} & =-K \gamma, \\
\hat{\mathbf{t}} \cdot \mathbf{T} \cdot \hat{\mathbf{n}} & =0,
\end{aligned}
$$

Copyright $\odot$ by SIAM. Unauthorized reproduction of this article is prohibited. 
where $\boldsymbol{u}_{I}=h_{t} \hat{\mathbf{k}}$ is the velocity of the interface, $\mathbf{T}$ is the Newtonian stress tensor given by

$$
\mathbf{T}=-p \mathbf{I}+\mu\left(\nabla \boldsymbol{u}+\nabla \boldsymbol{u}^{T}\right),
$$

$K$ is twice the mean curvature of the interface, $\gamma$ is the (assumed constant) surface tension, and $\hat{\mathbf{n}}$ and $\hat{\mathbf{t}}$ are the unit normal and tangential vectors to the interface

$$
\hat{\mathbf{n}}=\frac{\left(-h_{x}, 1\right)}{\left(1+h_{x}^{2}\right)^{1 / 2}}, \quad \hat{\mathbf{t}}=\frac{\left(1, h_{x}\right)}{\left(1+h_{x}^{2}\right)^{1 / 2}} .
$$

Finally, $h_{t}$ and $h_{x}$ denote differentiation of $h$ with respect to time $t$ and space $x$.

Boundary conditions at the ends of the domain are also needed. In the tear film, we consider the two basic conditions of fixed film height and fixed film curvature at the upper and lower lids $x= \pm L$. These are expressed as

$$
h( \pm L, t)=h_{0}, \quad h_{x x}( \pm L, t)=h_{x x}^{0},
$$

where $L$ is the constant half-length of the film and $h_{0}$ and $h_{x x}^{0}$ are constants. We use $L=14$, corresponding to the actual dimensional average distance between human eyelids of $1.008 \mathrm{~cm}$, and $h_{0}=9$ and $h_{x x}^{0}=4$ which were determined to be suitable values for a human eye (Braun and Fitt [3]). While those authors also considered boundary conditions in which the flux at the lids was specified, we consider only the conditions given above. Note that these conditions do allow flux of fluid at $x= \pm L$. Boundary conditions at $x= \pm L$ on the variables in the contact lens will not be needed in the present model. We use an initial condition that models the post-blink geometry of a tear film as a parabolic menisci at the two lids connected with a uniform thickness film in the interior of the domain. In dimensionless form (see the next section), this is

$$
h(x, 0)= \begin{cases}h_{\min }(0) & \text { if }|x| \leq L-\Delta x_{m}, \\ h_{\min }(0)+\Delta h_{m}\left[|x|-\left(L-\Delta x_{m}\right)\right]^{2} & \text { if }|x|>L-\Delta x_{m}\end{cases}
$$

where $h_{\min }(0), \Delta h_{m}$, and $\Delta x_{m}$ are parameters to be specified. We take them to be the same as those of Braun and Fitt, namely, $h_{\min }(0)=1, \Delta h_{m}=2$, and $\Delta x_{m}=2$. It is assumed in the application of these boundary conditions that the eyelids cover the ends of the contact lens.

An alternative to (11) is a condition that states that the fluid at the free surface is tangentially immobile, $\boldsymbol{u} \cdot \hat{\mathbf{t}}=0$. This condition was used by Braun and Fitt [3] as an effective model of the outer, lipid, layer of a human tear film. Unless otherwise noted, our numerical results will be based on the free surface condition (11); however, we include a derivation of the resulting evolution equation based on the tangentially immobile condition in Appendix B. Under certain conditions and interpretations of dimensionless parameters, the results in terms of the thin film evolution equation are the same.

2.1. Dimensionless equations. We introduce the dimensionless variables (following Braun and Fitt [3])

$$
h=d \bar{h}, \quad H=d \bar{H}, \quad u=U_{0} \bar{u}, \quad v=\epsilon U_{0} \bar{v}, \quad U=U_{0} \bar{U}, \quad V=\epsilon U_{0} \bar{V}, \quad p=\frac{\mu U_{0}}{l \epsilon^{2}} \bar{p},
$$

$$
x=l \bar{x}, \quad y=d \bar{y}, \quad t=\frac{l}{U_{0}} \bar{t},
$$


where $\epsilon=d / l$ represents a ratio of typical vertical to horizontal length scales, which for tear films is typically small, and $U_{0}$ is a typical velocity scale.

The resulting dimensionless equations are given below (dropping bars). In the liquid region $0<y<h(x, t)$,

$$
\begin{aligned}
\frac{\partial u}{\partial x}+\frac{\partial v}{\partial y} & =0 \\
\epsilon^{2} R e\left(\frac{\partial u}{\partial t}+u \frac{\partial u}{\partial x}+v \frac{\partial u}{\partial y}\right) & =-\frac{\partial p}{\partial x}+\epsilon^{2} \frac{\partial^{2} u}{\partial x^{2}}+\frac{\partial^{2} u}{\partial y^{2}}+G \sin \theta \\
\epsilon^{3} R e\left(\frac{\partial v}{\partial t}+u \frac{\partial v}{\partial x}+v \frac{\partial v}{\partial y}\right) & =-\frac{1}{\epsilon} \frac{\partial p}{\partial y}+\epsilon^{3} \frac{\partial^{2} v}{\partial x^{2}}+\epsilon \frac{\partial^{2} v}{\partial y^{2}}-G \cos \theta
\end{aligned}
$$

where the Reynolds number $R e$ and the dimensionless gravity parameter are defined by

$$
R e=\frac{U_{0} l}{\nu}, \quad G=\frac{\rho g d^{2}}{\mu U_{0}}
$$

and $\nu=\mu / \rho$ is the kinematic viscosity.

In the porous region $-H<y<0$,

$$
\begin{aligned}
\frac{\partial U}{\partial x}+\frac{\partial V}{\partial y} & =0 \\
U & =-D a \frac{\partial p}{\partial x}+D a G \sin \theta \\
\epsilon^{2} V & =-D a \frac{\partial p}{\partial y}-\epsilon D a G \cos \theta
\end{aligned}
$$

where the Darcy number $D a$ is defined by

$$
D a=\frac{k}{d^{2}} .
$$

The boundary condition on $y=-H$ is

$$
V=0 .
$$

The boundary conditions on $y=0$ are

$$
\begin{aligned}
& v\left(x, y=0^{+}, t\right)=V\left(x, y=0^{-}, t\right), \\
& u\left(x, y=0^{+}, t\right)=U\left(x, y=0^{-}, t\right)+\frac{\sqrt{D a}}{\alpha} \frac{\partial u}{\partial y}\left(x, y=0^{+}, t\right), \\
& p\left(x, y=0^{+}, t\right)=p\left(x, y=0^{-}, t\right) .
\end{aligned}
$$

The boundary conditions on $y=h(x, t)$ are

$$
\begin{aligned}
h_{t}+h_{x} u(x, h, t) & =v(x, h, t), \\
-p+2 \epsilon^{2} \frac{\left(v_{y}-h_{x} u_{y}\right)+\epsilon^{2}\left(h_{x}^{2} u_{x}-h_{x} v_{x}\right)}{\left(1+\epsilon^{2} h_{x}^{2}\right)} & =\frac{1}{C a}\left(\frac{h_{x}}{\left(1+\epsilon^{2} h_{x}^{2}\right)^{1 / 2}}\right)_{x}, \\
u_{y}+\epsilon^{2}\left(v_{x}+2 h_{x}\left(v_{y}-u_{x}\right)-h_{x}^{2} u_{y}\right)-\epsilon^{4} h_{x}^{2} v_{x} & =0,
\end{aligned}
$$

where we have defined the capillary number $C a$

$$
C a=\frac{\mu U_{0}}{\gamma \epsilon^{3}} .
$$

Copyright (c) by SIAM. Unauthorized reproduction of this article is prohibited. 
2.2. Lubrication theory: Thin film limit. We next examine the above system of equations in the thin film limit of $\epsilon \ll 1$.

In the liquid region $0<y<h(x, t)$,

$$
\begin{aligned}
\frac{\partial u}{\partial x}+\frac{\partial v}{\partial y} & =0 \\
0 & =-\frac{\partial p}{\partial x}+\frac{\partial^{2} u}{\partial y^{2}}+G \sin \theta, \\
0 & =-\frac{\partial p}{\partial y}-\epsilon G \cos \theta .
\end{aligned}
$$

Here we have at least momentarily retained all gravity terms, including the term $\epsilon G \cos \theta$ in the vertical component of the momentum equation. Note that when $\theta=0$, it is common to formally assume that the parameter $\epsilon G=O(1)$ as $\epsilon \rightarrow 0$ (e.g., Davis and Hocking [31,32]), while for the case when $\theta=\pi / 2$, it is common to formally assume that $G=O(1)$ as $\epsilon \rightarrow 0$ (e.g., Braun and Fitt [3]) so that gravity is retained in either case. As we are interested in both configurations, we retain both terms here with the note that the contribution from the term $\epsilon G$ will be small.

In the porous region $-H<y<0$,

$$
\begin{aligned}
\frac{\partial U}{\partial x}+\frac{\partial V}{\partial y} & =0 \\
U & =-D a \frac{\partial p}{\partial x}+D a G \sin \theta \\
0 & =-D a \frac{\partial p}{\partial y}-\epsilon D a G \cos \theta
\end{aligned}
$$

The boundary condition on $y=-H$ is

$$
V=0 .
$$

The boundary conditions on $y=0$ are

$$
\begin{aligned}
& v\left(x, y=0^{+}, t\right)=V\left(x, y=0^{-}, t\right), \\
& u\left(x, y=0^{+}, t\right)=U\left(x, y=0^{-}, t\right)+\frac{\sqrt{D a}}{\alpha} \frac{\partial u}{\partial y}\left(x, y=0^{+}, t\right), \\
& p\left(x, y=0^{+}, t\right)=p\left(x, y=0^{-}, t\right) .
\end{aligned}
$$

The boundary conditions on $y=h(x, t)$ are

$$
\begin{aligned}
h_{t}+h_{x} u(x, h, t) & =v(x, h, t), \\
-p & =\frac{1}{C a} h_{x x}, \\
u_{y} & =0 .
\end{aligned}
$$

Using these equations and boundary conditions, we can write expressions for $u, v$, $U, V$, and $p$ in the liquid and porous regions. The velocity components in the liquid are

$$
u(x, y, t)=\left(-\frac{1}{C a} \frac{\partial^{3} h}{\partial x^{3}}+\epsilon G \cos \theta \frac{\partial h}{\partial x}-G \sin \theta\right)\left[\frac{1}{2} y^{2}-h y-\frac{\sqrt{D a}}{\alpha} h-D a\right]
$$




$$
\begin{aligned}
v(x, y, t)= & \left(\frac{1}{C a} \frac{\partial^{4} h}{\partial x^{4}}-\epsilon G \cos \theta \frac{\partial^{2} h}{\partial x^{2}}\right)\left[\frac{1}{6} y^{3}-\frac{1}{2} h y^{2}-\frac{\sqrt{D a}}{\alpha} h y-D a(y+H)\right] \\
& +\left(-\frac{1}{C a} \frac{\partial^{3} h}{\partial x^{3}}+\epsilon G \cos \theta \frac{\partial h}{\partial x}-G \sin \theta\right)\left(\frac{1}{2} y^{2}+\frac{\sqrt{D a}}{\alpha} y\right) \frac{\partial h}{\partial x}
\end{aligned}
$$

The volume flow rate components in the porous region are

$$
\begin{aligned}
U(x, t) & =\frac{D a}{C a} \frac{\partial^{3} h}{\partial x^{3}}-\epsilon D a G \cos \theta \frac{\partial h}{\partial x}+D a G \sin \theta, \\
V(x, y, t) & =-\left[\frac{D a}{C a} \frac{\partial^{4} h}{\partial x^{4}}-\epsilon D a G \cos \theta \frac{\partial^{2} h}{\partial x^{2}}\right](y+H) .
\end{aligned}
$$

The pressure in both the liquid and porous regions has the same form given by

$$
p(x, t)=-\frac{1}{C a} \frac{\partial^{2} h}{\partial x^{2}}-\epsilon(y-h) G \cos \theta .
$$

These forms satisfy (34)-(36), (37)-(39), and boundary conditions (40), (41)-(43), and (45)-(46). The only boundary condition that remains to be satisfied is the kinematic condition (44).

Substituting $u(x, y, t)$ and $v(x, y, t)$ evaluated at $y=h$ into boundary condition (44) leads to the evolution equation for the interface position $h(x, t)$

$$
\frac{\partial h}{\partial t}=-\frac{\partial}{\partial x}\left[F(h)\left(\frac{1}{C a} \frac{\partial^{3} h}{\partial x^{3}}-\epsilon G \cos \theta \frac{\partial h}{\partial x}+G \sin \theta\right)\right],
$$

where $F(h)$ may have the following forms, depending on which particular choice for slip boundary condition (Beavers-Joseph or Le Bars-Worster) at the liquid-porous interface is used

$$
\begin{aligned}
F_{B J} & =\frac{1}{3} h^{3}+\frac{\sqrt{D a}}{\alpha} h^{2}+D a(h+H), \\
F_{L W} & =\frac{1}{3}(h+\delta)^{3}+D a(h+H) .
\end{aligned}
$$

The details of the derivation of the evolution equation for the Le Bars-Worster condition are given in Appendix A. In the absence of slip (take $1 / \alpha=0$ in $F_{B J}$ or $\delta=0$ in $\left.F_{L W}\right)$, one recovers a "standard" no-slip case, where $F(h)=\frac{1}{3} h^{3}+D a(h+H)$. When the tangentially immobile condition is applied at the fluid-air interface, we obtain evolution equations of the same general form as given here but with different forms for the function $F(h)$ given by $F_{B J T I}$ and $F_{L W T I}$ as outlined in Appendix B. A straightforward linear stability analysis of a planar interface solution to this equation (see Appendix C) shows that the detailed form of the different functions $F$, for different slip models and interfacial conditions, influences the decay rate of the infinitesimal perturbations, but the parameters $D a, \alpha$, and $\delta$ representing permeability and slip are not themselves a source of instability for a planar interface.

It is instructive to make some comparisons of our thin film evolution equation to existing thin film evolution equations. We first note that in the limit $D a \rightarrow 0$, our coupled fluid-porous model reduces to a fluid layer on an impermeable boundary. In this case, with $D a=0$ and no slip, we find that $F(h)=\frac{1}{3} h^{3}$. This form for $F(h)$ and the evolution equation is in agreement with the results of Greenspan [43] 
(in the absence of gravity) and Hocking [44] (with gravity term $\epsilon G$ neglected) if one neglects slip on the fluid-solid boundary in those models. Both Greenspan's model and Hocking's model were applied to problems with contact lines and so included slip at the fluid-solid boundary. Greenspan implemented a slip condition at a fluid-solid boundary of the form $u=\beta(h) \partial u / \partial z$ with $\beta(h)=\beta_{1} / h$, where $h$ is the thickness of the fluid layer and $\beta_{1}$ a constant, and obtained $F(h)=\frac{1}{3} h^{3}+\beta_{1} h$. Hocking [44] implemented a slip condition with constant $\beta(h)=\beta_{0}$ and obtained $F(h)=$ $\frac{1}{3} h^{3}+\beta_{0} h^{2}$. The effect of fluid-solid slip on $F(h)$ in the Greenspan or Hocking model is similar to the effect of fluid-porous slip as well as simply the presence of the porous layer on $F(h)$ in our model. In particular, a slip-related term proportional to $h^{2}$ with coefficient $D a / \alpha$ appears in our $F_{B J}$. The slip parameter $\delta$ of the Le Bars-Worster model gives rise to slip terms with powers $h^{2}$ and $h$, as well as a term independent of $h$ [expanding $(h+\delta)^{3}$ in $F_{L W}$ ]. However, even in the absence of slip on the fluidporous interface (i.e., $1 / \alpha=0$ or $\delta=0$ ), we observe terms proportional to $h$ and terms independent of $h$ that arise due to the presence of the porous base. We shall show that, consistent with thin film theory of Bertozzi et al. [45] and Bertozzi [46], the presence of these constant terms in $F(h)$ has important consequences on the rupture dynamics of the film. As pointed out by Devauchelle, Josser, and Zaleski [33] a similar form for $F(h)$ obtained for the case of a porous base with Beavers-Joseph slip is also obtained if one implements a second-order slip condition [47, 48] at a fluid-solid boundary.

Our equations reflect the impermeable boundary at the bottom of the porous layer. Models that address draining through an initially dry porous layer (e.g., Acton, Huppert, and Worster [34] and Davis and Hocking [32]) have another free boundary in the porous medium, as well as a different vertical velocity at the fluid-porous boundary, and consequently lead to a somewhat different form for the free surface evolution equation than that given here.

We can compare our equation to that of Braun and Fitt [3] in the basic case with zero gravity and evaporation in their model and $D a=0$, no slip, and zero gravity in our model. In this case we note that even though Braun and Fitt used the tangentially immobile boundary condition at the free surface while we have implemented a stressfree interface condition, we can recover their result by simply modifying the values of $C a$ and $G$ used here. In this case we find $F(h)=\frac{1}{3} h^{3}$ for the stress-free condition and $F(h)=\frac{1}{12} h^{3}$ for the tangentially immobile case. That is, the tangentially immobile case of Braun and Fitt with $C a=1$ and $G=1$ is equivalent to that obtained from the evolution equation with the stress-free boundary in the absence of the porous layer when $C a=4$ and $G=1 / 4$. Therefore, in the absence of a porous layer, this can be translated into a factor of four change in time scale; that is, the dynamics in the tangentially immobile case are four times slower than that for the stress-free case. When the effects of slip and the porous layer are included, the relationship between the function $F(h)$ in cases with either stress-free or tangentially immobile boundary conditions are more complicated, as is shown in Appendix B. However, we find that for the Beavers-Joseph slip and the Le Bars-Worster slip under the conditions that $D a \ll 1, \delta \ll 1$, and $H \gg 1$, the function $F(h)$ takes the forms

$$
\begin{aligned}
& F_{B J T I} \approx \frac{1}{4}\left[\frac{1}{3} h^{3}+\frac{\sqrt{D a}}{\alpha} h^{2}+4 D a H\right]+\ldots, \\
& F_{L W T I} \approx \frac{1}{4}\left[\frac{1}{3}(h+\delta)^{3}+4 D a H\right]+\ldots
\end{aligned}
$$

Therefore, a tangentially immobile case with $C a=1, G=1$, and a given value 
$H=H^{*}$ (i.e., using either $F_{B J T I}$ or $F_{L W T I}$ ) corresponds approximately to the case $C a=4, G=1 / 4$, and $H=4 H^{*}$ in the stress-free case (i.e., using either $F_{B J}$ or $F_{L W}$ ).

As noted above, equations of the form (52) are common in the description of hydrodynamic effects occurring in thin fluid films. Such equations have been the subject of theoretical analyses and numerical computation exploring the details of singularity formation and film rupture (Bertozzi et al. [45], Bertozzi [46]). Bertozzi and coworkers, in particular, explored a thin film equation of the form

$$
\frac{\partial h}{\partial t}=-\frac{\partial}{\partial x}\left(h^{n} \frac{\partial^{3} h}{\partial x^{3}}\right)
$$

for different values of the exponent $n$. Their work examined a variety of boundary conditions, including "pressure" boundary conditions $\left[h( \pm 1)=1\right.$ and $h_{x x}( \pm 1)=p$, where $p$ is a given constant] which correspond to the ones used in our work. They were able to classify predicted and observed singular rupture behavior $(h \rightarrow 0)$ in terms of the exponent $n$ and the constant $p$. One of their results (see Corollary 6.1.1 in Bertozzi et al. [45]) states that a solution $h$ to (57) with smooth initial data and the above pressure conditions with $p>2$ will always go to zero in either finite or infinite time. They also found that finite time singularities tend to be favored for smaller values of $n$. In particular, for these "pressure" boundary conditions, finite time singularities are not possible when $n \geq 4$ (or $n \geq 2$, provided $h_{x x}$ remains bounded). Further, their analyses and simulations showed that for values of $n$ in the range $0 \leq n \leq 1.2$, finite time singularities were observed, while for $n$ approximately greater than 0.75 (indicating some overlap with the above interval), infinite time singularities were observed (see Table 1 in [45]).

Two basic observations allow us to apply the predictions of Bertozzi et al. [45] to the rupture dynamics expected in our tear film model. First, our boundary conditions at $x= \pm L$ can be expressed in the form of the "pressure" boundary conditions considered by Bertozzi et al. with $p=4(14)^{2} / 9 \approx 87>2$. Second, our function $F(h)$ in (52), defined specifically for the various models and boundary conditions by (53), (B4), (54), and (B9), has the form $F(h) \sim h^{n}$ in the limit $h \rightarrow 0$. Therefore, based on the theory of Bertozzi et al., we can conclude that rupture will always occur in our tear film model. Further, the limit $h \rightarrow 0$ in our (53), (B4), (54), and (B9) reveals that four different values of the exponent $n(=0,1,2,3)$ may arise. First, the value $n=3$ follows from either (53) or (B4) with $D a=0$ and from either (54) or (B9) with $D a=0$ and $\delta=0$. These correspond to the well-known case of thin film flow over an impermeable no-slip boundary. Next, and more importantly for the porous substrate/contact lens model, the exponent $n=0$ is obtained whenever $D a$ and $H$ are nonzero in (53), (B4), (54), or (B9), that is, for any case with a porous substrate/lens of nonzero thickness. Furthermore, at least formally, one obtains the case $n=0$ in (54) and (B9) when $D a=0$ and $\delta \neq 0$, although based on the nature of the Le Bars-Worster slip model, one would expect to use $\delta=0$ when $D a=0$. The case $n=1$ is formally obtained when $D a \neq 0$ and $H=0$ in (53) or when $D a \neq 0$ and $\delta=H=0$ in (54) and (B9). Finally, the case $n=2$ is obtained when $D a \neq 0$ and $H=0$ in (B4).

As noted above, the theory of Bertozzi et al. [45] implies that tear film rupture will always occur in our model. Their theory also indicates that we should see finite time singularities when a porous substrate is present $(n=0)$ and infinite time singularities when the porous substrate is not present $(n=3)$. For application to the tear film problem, the details of any finite time singularities need to be compared with other 
relevant time scales in our problem - e.g., typical time between blinks. However, at least in theory, the difference between finite and infinite time singularities is significant, and the presence of terms in $F(h)$ associated with $D a H$ or $\delta$, however small, in principle makes a fundamental change to the dynamics. We shall quantify these observations in more detail in the results given below.

3. Results. The thin fluid film evolution equation (52) is a fourth-order nonlinear partial differential equation. In order to obtain results showing the effects of the presence of the porous layer (with associated parameters Darcy number $D a$ and lens thickness $H$ ), the variation of slip at the liquid/porous interface $(1 / \alpha$ and $\delta)$, and the film orientation factors ( $\theta$ and gravity $G$ ), we solve (52), (53), and (54) numerically.

3.1. Numerical method and parameter estimation. We use a method of lines approach to solve these equations. In particular, we use a second-order accurate conservative finite difference scheme in space to discretize the equations at $N+1$ equally spaced points on the spatial domain and to generate a system of ordinary differential equations which we solve numerically using MATLAB's ode23s solver. For further details on numerical methods for this type of thin film evolution equation, see, for example, Beerman and Brush [49], Bertozzi et al. [45], and Bertozzi [46]. All computations were performed on the space domain of $-L \leq x \leq L$ with boundary values as described in the previous section.

The following results use a discretization with $N=2000$ which, by numerical and graphical comparison with results with less resolution (e.g., $N=500$ and $N=1000$ ), we have determined provides sufficient accuracy to interpret the results presented. Our numerical results were also validated by comparing them with the zero gravity, no evaporation case of Braun and Fitt (private communication with R. J. Braun [50]). This was accomplished by using parameter values $C a=4, D a=0, G=0$, and, in the case of the Le Bars-Worster slip condition, $\delta=0$. (Note that when $D a=0$, the values of $1 / \alpha$ and $H$ do not affect the solution.) Our emphasis in this work will be on the effects of the new terms that relate directly to the contact lens model. In particular, we are primarily interested in the influence of the Darcy number $D a$ (which is a measure of the pore scale radius of the lens relative to a typical fluid layer thickness), the contact lens thickness $H$, the slip condition at the film-lens interface as measured by $1 / \alpha$ or $\delta$, and gravitation effects measured by $G$. In our calculations, we have focused primarily on functions $F_{B J}$ and $F_{L W}$. As pointed out above, however, interpretations of our results applicable for functions $F_{B J T I}$ and $F_{L W T I}$ for tangentially immobile cases can be obtained by appropriate reinterpretation of the parameters. The results below highlight the effect of these model parameters on the film profile and dynamics.

In order to determine typical values for our dimensionless lens thickness $H$, we note that this is the ratio of the actual contact lens thickness relative to a typical tear film thickness. The contact lenses used in the study by Fornasiero, Prausnitz, and Radke [26] were reported to have thickness of $100 \mu \mathrm{m}$ when water saturated. Raad and Sabau [23] used a lens thickness value of $300 \mu \mathrm{m}$ in their study of contact lens dynamics during blinking. The soft contact lenses examined in the clinical studies of Maldonado-Codina and Efron [20] range from 60 to $110 \mu \mathrm{m}$. The soft contact lenses examined by Monticelli, Chauhan, and Radke [24] had thickness of $200 \mu \mathrm{m}$. If we take as a typical tear film thickness between 1 and $5 \mu \mathrm{m}$ (e.g., see Bron et al. [5] or Nichols and King-Smith [16]) and use a representative contact lens thickness of $100 \mu \mathrm{m}$, then typical values for $H$ range between 20 and 100. Measurements of the lens permeability by Monticelli, Chauhan, and Radke [24] indicate that a realistic value for $D a$ for soft contact lenses is $D a=10^{-8}$ (based on their measured permeability of approximately 
$10^{-8} \mu \mathrm{m}^{2}$ and a tear film thickness of $1 \mu \mathrm{m}$ ) or even smaller. Our results will show that this relatively small value of $D a$ will lead to only minor changes in the tear film dynamics over time scales between blink cycles. As has been done in previous studies (e.g., Raad and Sabau [23]), however, it will be instructive in certain cases to show results for larger values of $D a$ to highlight features of the model associated with the presence of a porous substrate. For the dimensionless Beavers-Joseph slip parameter $\alpha$, we note that Raad and Sabau [23] used the same parameter with values 0.3 and 10. For completeness and lack of specific measurements for slip effects, we consider a broad range of values for $\alpha$ and $\delta$.

The time scale used in the nondimensionalization is given by $l / U_{0}$. Estimates for these parameters obtained from Braun and Fitt [3], based on a typical film thickness of $10 \mu \mathrm{m}$, are $l=0.36 \mathrm{~mm}$ and $U_{0}=0.75 \mathrm{~mm} \mathrm{~s}^{-1}$ which gives a time scale of approximately $0.5 \mathrm{~s}$. Using $5 \mu \mathrm{m}$ instead, which is more in line with current thinking on the tear film thickness, we find that $l=0.28 \mathrm{~mm}$ and $U_{0}=0.19 \mathrm{~mm} \mathrm{~s}^{-1}$, giving a time scale of approximately $1.5 \mathrm{~s}$. Smaller film thickness values lead to still longer time scales. In the calculations that follow, we have computed solutions out to a dimensionless time of 100 or further. Using the two estimates above, a dimensionless time of 100 corresponds to either 50 or $150 \mathrm{~s}$. While a typical time between blinks is less than $10 \mathrm{~s}$, this value can vary in either direction, depending on the individual and the activity. The dimensionless times we have used in our calculations are presumably sufficient to capture important scales of interest for typical contact lens wear, as well as to demonstrate novel long time aspects of the mathematical model.

3.2. Influence of lens permeability. In Figure 3 we show film profiles at two different times with and without a porous layer. Here we do not include the effects of slip (i.e., $1 / \alpha=0$ and $\delta=0$ ). The case $D a=0$ corresponds to a tear film without a contact lens (i.e., a fluid film on an impermeable boundary), and $D a \neq 0$ corresponds to the presence of a contact lens. Note that when $D a=0$, the boundary $y=0$ represents the corneal surface, while when $D a \neq 0$, this boundary represents the contact lens surface. The predictions for the tear film dynamics with and without a contact lens are nearly indistinguishable over the time scales shown when $D a=10^{-8}$.

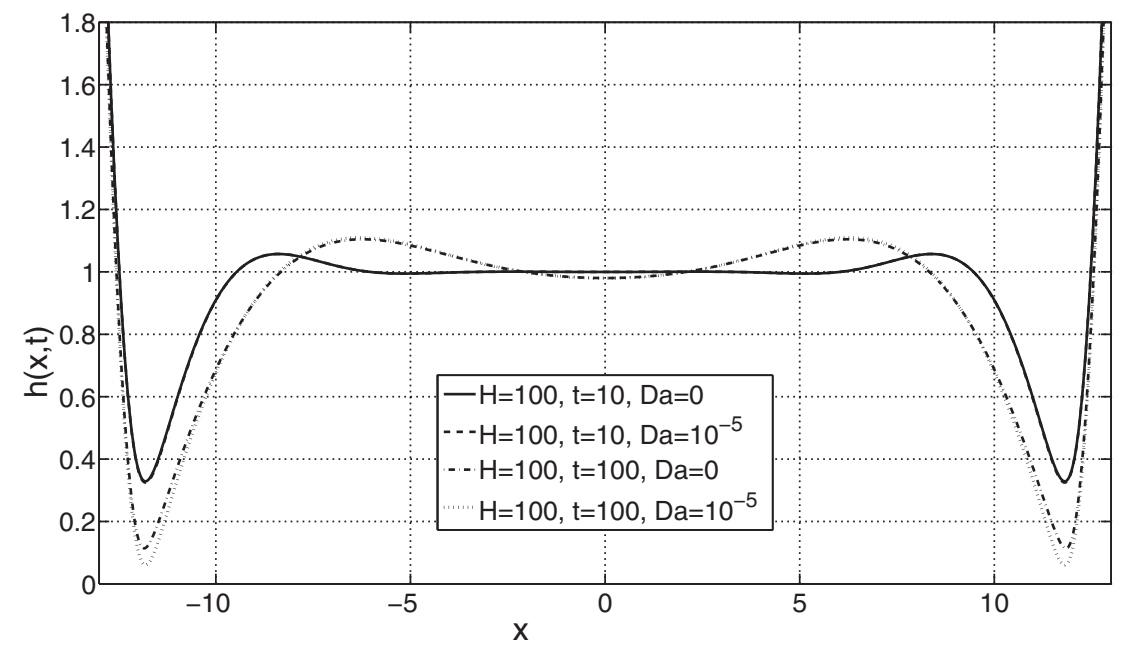

FIG. 3. Fluid film profiles for two different Darcy numbers $\left(D a=0\right.$ and $\left.D a=10^{-5}\right)$ at two different times $(t=10$ and $t=100)$ for a fixed value of $H=100$. Here $1 / \alpha=0$ and $\delta=0$. 


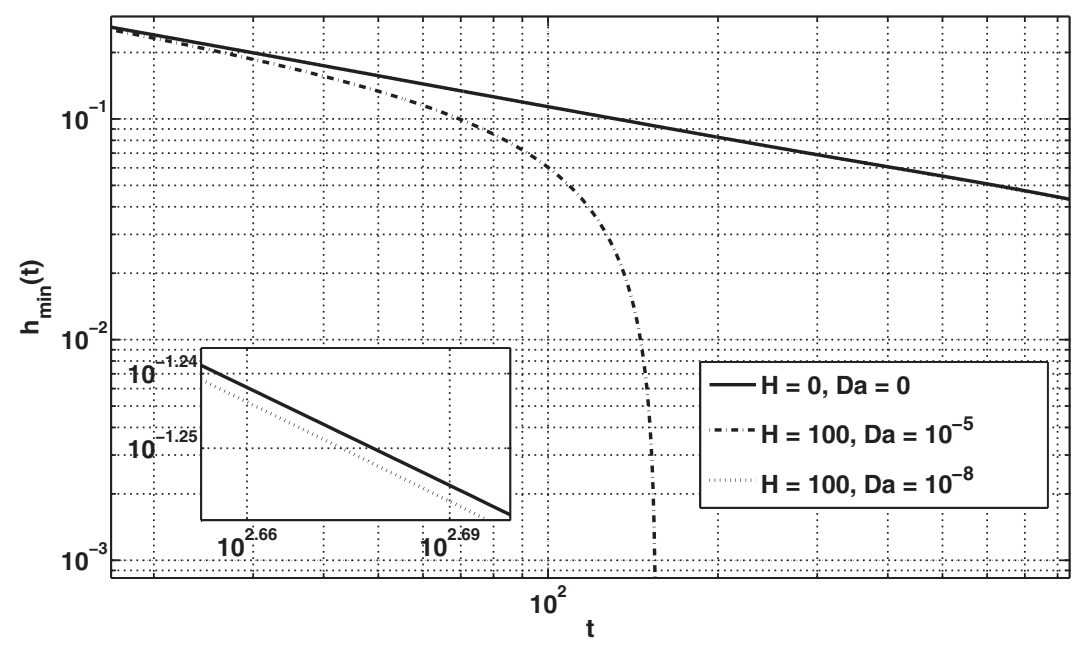

FIG. 4. Minimum film thickness as a function of time for different values of Darcy number $\left(D a=10^{-8}\right.$ and $\left.10^{-5}\right)$ for $H=100$. Also shown for reference is the case with $D a=0$ and $H=0$ representing a fluid film on a solid boundary (i.e., no porous layer). Here $1 / \alpha=0$ and $\delta=0$.

We have shown here a comparison of the tear film dynamics for a significantly larger value of $D a=10^{-5}$ to demonstrate that the presence of the contact lens enhances thinning slightly. In either case, the film thins most dramatically in the "black line" that separates the meniscus near the lids from the central portion of the tear film. We quantify this film thinning for a range of $D a$ in more detail in the next figure.

In Figure 4 we show the effect of the porous layer/contact lens on the minimum tear film thickness. The curve with $D a=0$ is the uppermost curve and corresponds to the dynamics of a tear film in the absence of a contact lens. This case is the basic case (no evaporation, no gravity) outlined in Braun and Fitt [3]. The case with $D a=10^{-8}$ and $H=100$, which is nearly coincident with the $D a=0$ result, shows that for time scales up to $t=500$, there is only a minimal effect on the thinning dynamics; the inset graph shows a close-up of the difference between these two cases at the far right of the main graph. A third curve, with $D a=10^{-5}$, shows more clearly that the porous substrate enhances thinning of the fluid and can, in fact, lead to rupture of the film in finite time, consistent with the theory of Bertozzi et al. [45] and Bertozzi [46]. We explore further the possibility of finite time rupture later in this section but conclude here that for realistic permeability values and for typical time scales between blinks, the influence of permeability on the tear film dynamics during contact lens wear is minor.

3.3. Influence of lens thickness. We next examine the influence of contact lens thickness $H$ on the film rupture dynamics. Here we fix the value of Darcy number $D a=10^{-8}$ and neglect gravitational and slip effects. Figure 5 shows the minimum tear film thickness as a function of time for a range of typical values of $H$. Again for reference we show the minimum film thickness curve corresponding to the absence of a contact lens (solid line); this is the same as the solid curve in Figure 4. While the overall differences between the cases with and without a contact lens are small, the trend of increasing contact lens thickness is clear; increasing the contact lens thickness increases the rate at which the tear film ruptures. Recall that the effect of $H$ in the tangentially immobile case would be more pronounced than that shown here. For example, the curve with $H=100$ in Figure 5 would correspond to $H=25$ in the 


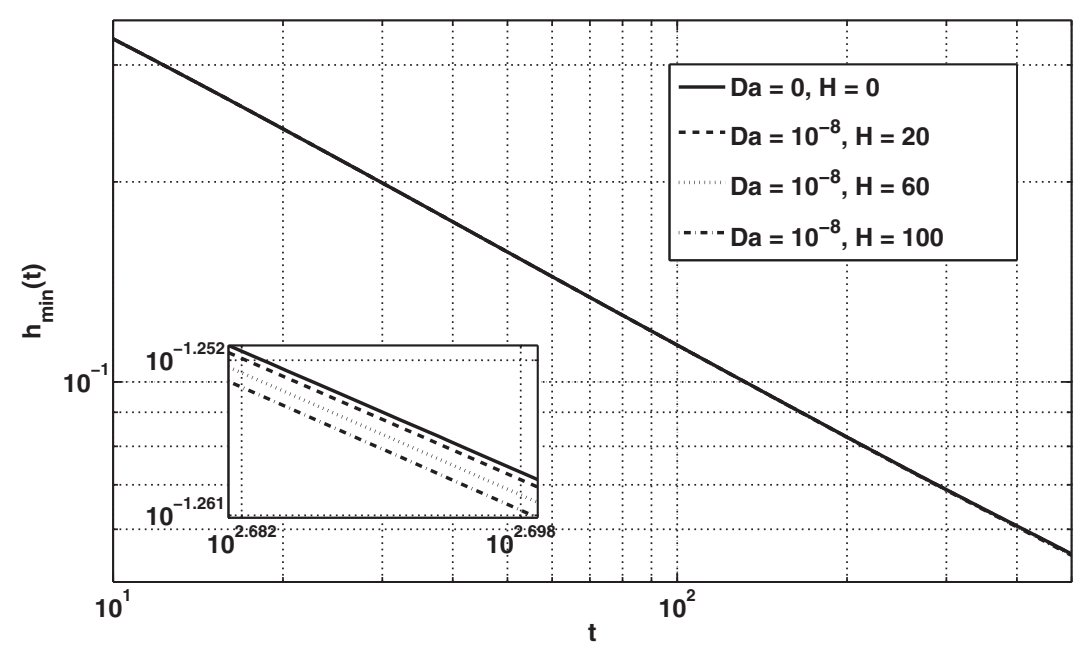

Fig. 5. Minimum film thickness as a function of time for different values of porous layer thickness $\left(H=20,60\right.$, and 100) for $D a=10^{-8}$. Also shown for reference is the case with $H=0$ and $D a=0$ representing a fluid film on a solid boundary (i.e., no porous layer). Here $1 / \alpha=0$ and $\delta=0$.

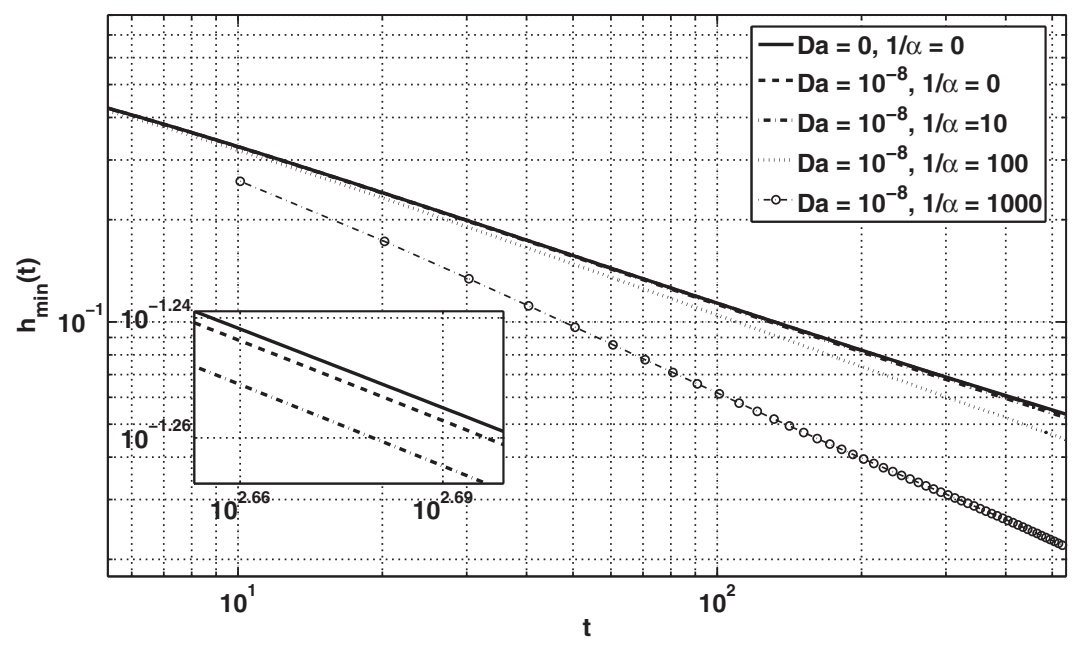

Fig. 6. Minimum film thickness as a function of time for different values of the Beavers-Joseph slip parameter $1 / \alpha\left(1 / \alpha=0,10,100\right.$, and 1000) for $D a=10^{-8}$ and $H=100$. Also shown for reference is the case with $1 / \alpha=0$ and $D a=0$ representing a fluid film on a solid boundary (i.e., no porous layer) with no slip.

tangentially immobile case. We presume that as these results correspond to the case $n=0$ in the notation of Bertozzi et al. [45] and Bertozzi [46], the expected finite time rupture occurs beyond the final time shown in the figure.

3.4. Influence of slip. In the next two figures we explore the effects of two different models of slip at the fluid-porous interface. First, we look at the effect of the Beavers--Joseph slip boundary condition which corresponds to the evolution equation (52) and the function $F_{B J}$ given by (53). Note that the dimensionless slip length in this model is $\sqrt{D a} / \alpha$. Figure 6 shows the minimum film thickness as a 


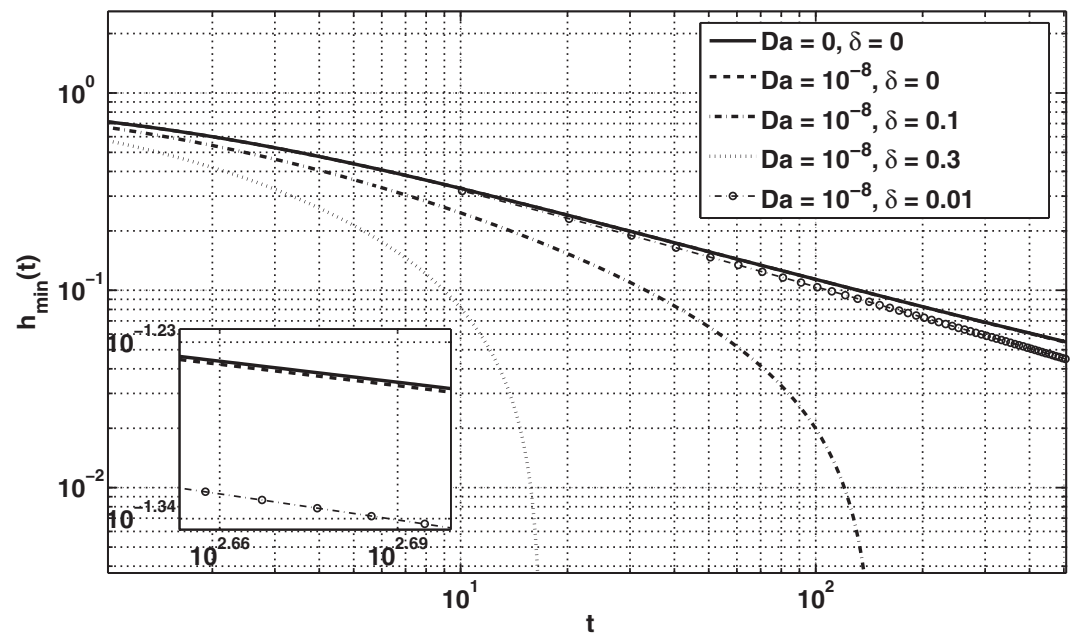

FIG. 7. Minimum film thickness as a function of time for different values of the Le BarsWorster slip parameter $\delta(\delta=0,0.01,0.1$, and 0.3$)$ for $D a=10^{-8}$ and $H=100$. Also shown for reference is the case with $\delta=0$ and $D a=0$ representing a fluid film on a solid boundary (i.e., no porous layer) with no slip.

function of time for three different nonzero values of the slip parameter $1 / \alpha$. We have chosen a broad range of values in order to demonstrate the general effect of the Beavers-Joseph slip condition. Again for reference we have shown the basic no slip, no contact lens case by the solid line. Also shown for reference is the case with a contact lens with no slip (dashed line). The case $1 / \alpha=10$, which corresponds to a dimensionless slip length of $0.1 \%$ of the typical tear film thickness, is shown by the dashed-dotted curve. The case $1 / \alpha=100$, which corresponds to a case of slip of $1 \%$ of the typical tear film thickness, is shown by the dotted curve. Finally, the case $1 / \alpha=$ 1000 , which corresponds to a case of slip of $10 \%$ of the typical tear film thickness, is shown by the dashed-circle curve. We observe that the inclusion of slip leads to slightly faster film thinning. We note that while the cases with $D a \neq 0$ and $H \neq 0$ in this figure technically correspond to $n=0$ in the Bertozzi et al. $[45,46]$ theory, we do not observe finite time rupture over this time interval. We can understand this by noting that for these values of $h_{\min }, D a$, and $H$, the term with $n=3$ is still orders of magnitude larger than the $n=0$ term. Also, again for these values of $h_{\min }$, the $n=2$ term is larger than the $n=0$ term and may be comparable to the $n=3$ term, depending on the value of $1 / \alpha$.

Next, we study the Le Bars-Worster slip boundary condition which allows slip into the porous layer. Figure 7 shows the minimum tear film thickness as a function of time for different values of $\delta$. For reference, we have shown the basic no-slip, no contact lens case (solid line), as well as the (nearly indistinguishable) case with a contact lens with no-slip (dashed line). The case $\delta=0.01$, which corresponds to a dimensionless slip depth (into the porous lens) of $1 \%$ of the typical tear film thickness, is shown by the dashed-circle curve. The case $\delta=0.1$, which corresponds to a dimensionless slip depth of $10 \%$ of the typical tear film thickness, is shown by the dashed-dotted curve. The case $\delta=0.3$, which corresponds to a relatively extreme case of slip of $30 \%$, is shown by the dashed curve. The two cases shown with the largest nonzero $\delta(0.1$ and 0.3 ) show that, consistent with the Bertozzi et al. $[45,46]$ theory with $n=0$, finite 


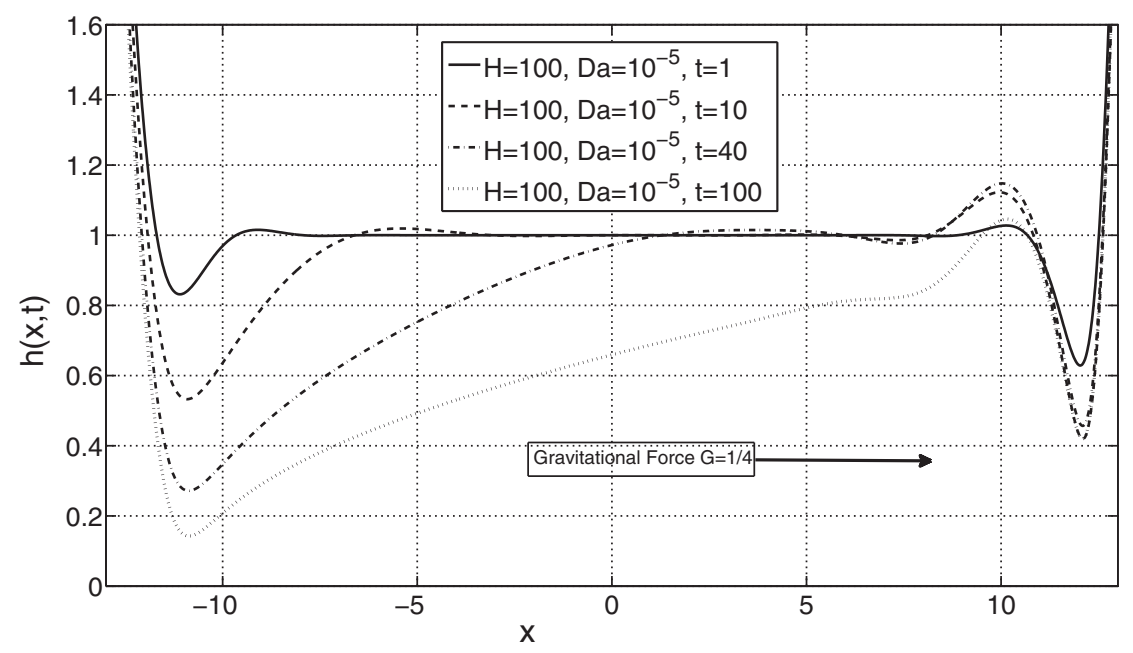

FIG. 8. Fluid film profiles for nonzero gravity (gravitational force along the $x$ direction with $G=1 / 4$ ) at four different times $\left(t=1,10,40\right.$, and 100), while using a fixed value of $D a=10^{-5}$, $H=100, \theta=\pi / 2$, and no slip.

time rupture occurs. The case with $\delta=0.01$, which technically corresponds to $n=0$ as $h \rightarrow 0$, has not yet reached that asymptotic regime; for these values of $h_{\min }$, the $n=3$ term in $F_{L W}$ is still the dominant term. In general, we note that slip leads to increased film thinning.

3.5. Influence of gravity. Finally, we study the tear film evolution with and without gravity (i.e., $G=1 / 4$ and $G=0$, respectively) with film orientation $\theta=\pi / 2$. Figure 8 shows the time evolution of the film for a value $G=1 / 4$ consistent with the value used by Braun and Fitt [3]. We observe that for short times, the minimum film thickness occurs at the bottom of the eye $(x=L$ on the right side of the plot). However, for later times, the gravitational drainage dominates and leads to a film whose thinnest part is near the top of the eye $(x=-L$ on the left side of the plot). This is the same general behavior observed by Braun and Fitt [3]. We examine this result further in Figure 9 to clarify the role of gravity with and without contact lenses.

In Figure 9 we show the minimum film thickness as a function of time for the case with fixed $H$, no slip, and two different Darcy numbers with and without the inclusion of gravity. As we observed previously for the case of zero gravity, the effect of increasing $D a$ in general promotes film thinning. This is also the case when gravity is nonzero. When gravity is zero, the film thins symmetrically. However, when gravity is nonzero with $\theta=\pi / 2$, this symmetry is broken. As was noted in the previous figure, at early times the minimum film thickness occurs at the bottom of the tear film (lower eye lid), but a transition occurs (between $t=10$ and 20) in which gravitation drainage causes increased film thinning near the upper eye lid, resulting in the minimum film thickness shifting to the upper portion of the tear film.

3.6. Finite versus infinite time rupture. In this section we present two additional studies that can be interpreted in terms of the Bertozzi et al. [45, 46] theory in which finite or infinite time rupture can be predicted on the basis of the exponent $n$, where $F(h) \rightarrow h^{n}$ as $h \rightarrow 0$. As noted above, the theory and computational results of Bertozzi et al. [45, 46] predict that for "pressure" boundary conditions with $p>2$ 


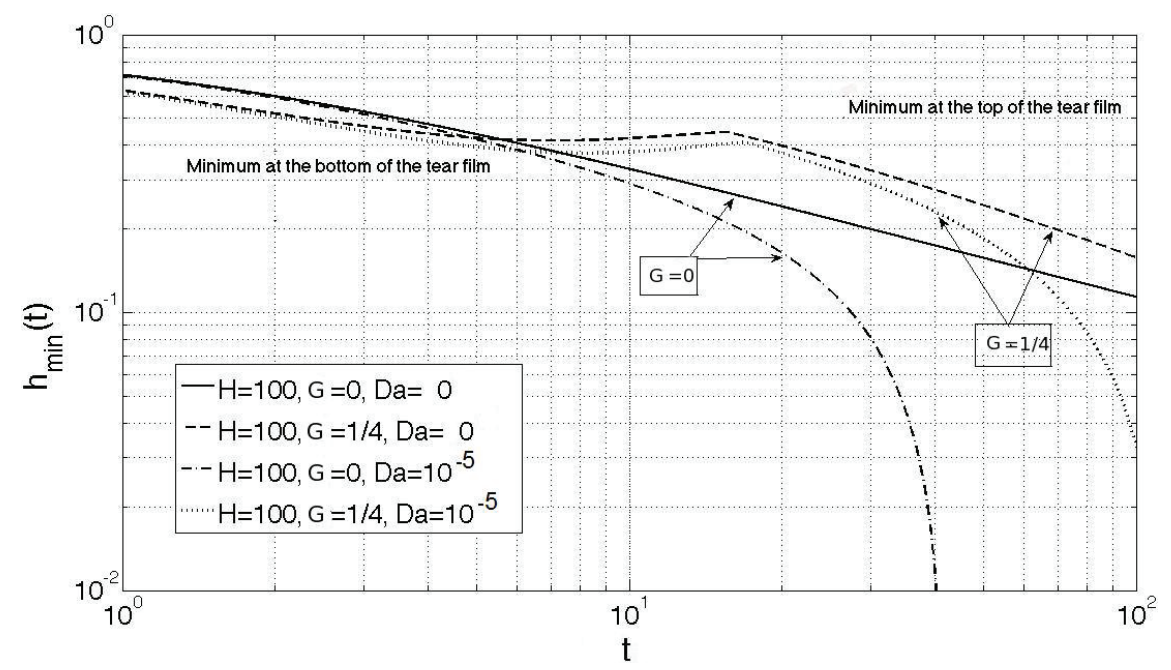

FIG. 9. Minimum film thickness as a function of time for two different Darcy numbers $(D a=0$ and $\left.D a=10^{-5}\right)$ for a fixed value of $H=100$ with and without gravity $(G=0$ and $1 / 4)$ for $\theta=\pi / 2$ and no slip.

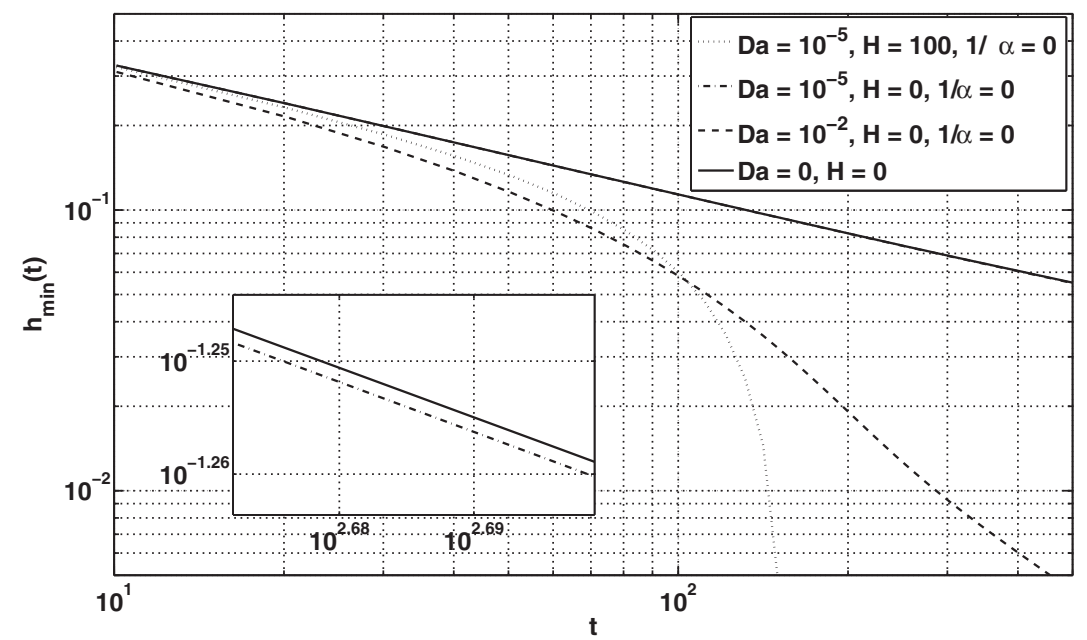

FIG. 10. Minimum film thickness as a function of time for cases corresponding to three different values of $n$, where $F(h) \rightarrow h^{n}$ as $h \rightarrow 0$. In particular, $F_{B J}$ with $D a=0(n=3)$; $F_{B J}$ with $D a=10^{-5}, 1 / \alpha=0$, and $H=0(n=1) ; F_{B J}$ with $D a=10^{-2}, 1 / \alpha=0$, and $H=0$ (also $\left.n=1\right)$; and $F_{B J}$ with $D a=10^{-5}, 1 / \alpha=0$, and $H=100(n=0)$.

equivalent to those considered here (our $p$ is approximately 87), values of $n$ in the range $0 \leq n \leq 1.2$ corresponded to finite time singularities in the film dynamics, while values of $n$ approximately greater than 0.75 corresponded to infinite time singularities.

In Figure 10 we explore the Beavers-Joseph boundary condition for scenarios in which $F_{B J}(h) \rightarrow h^{n}$ as $h \rightarrow 0 ; D a=0(n=3) ; D a \neq 0,1 / \alpha=0$, and $H=0(n=1)$, and $D a \neq 0,1 / \alpha=0$, and $H \neq 0(n=0)$. The results of Bertozzi et al. [45, 46] suggest that the $n=3$ case, which here corresponds to thin film evolution on an impermeable substrate (e.g., no contact lens), should follow dynamics in which rupture occurs in 


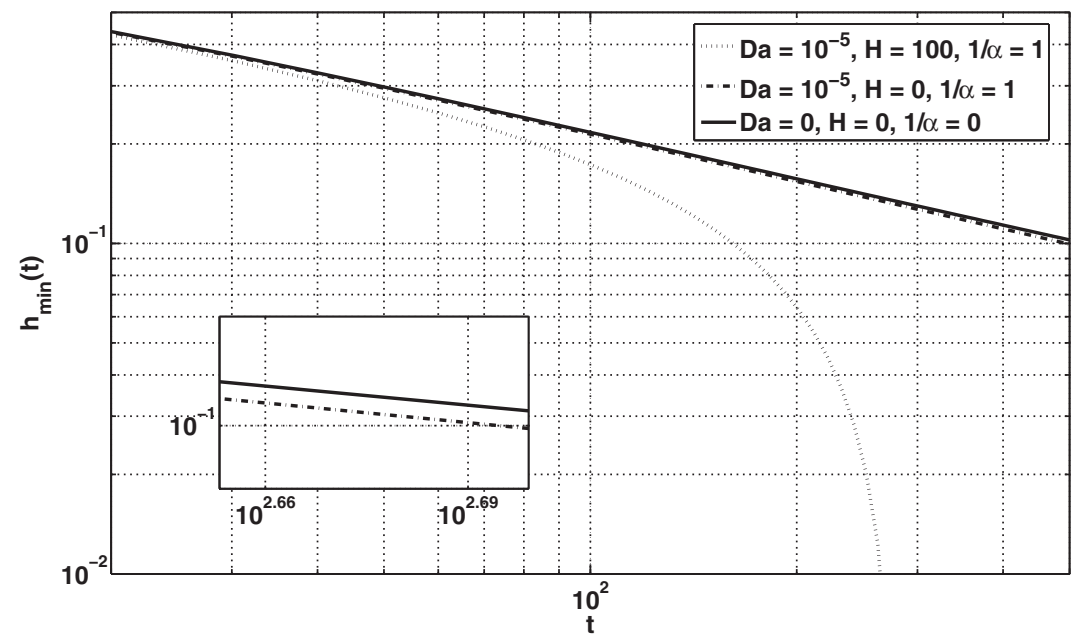

FIG. 11. Minimum film thickness as a function of time for three cases corresponding different values of $n$ where $F(h) \rightarrow h^{n}$ as $h \rightarrow 0$. In particular, $F_{B J T I}$ with $D a=0(n=3)$; $F_{B J T I}$ with $D a=10^{-5}, 1 / \alpha=1$, and $H=0(n=2) ;$ and $F_{B J T I}$ with $D a=10^{-5}, 1 / \alpha=1$, and $H=100$ $(n=0)$.

infinite time. For the $n=0$ case, corresponding here to the presence of a porous substrate, the Bertozzi et al. [45, 46] theory predicts rupture in finite time. Figure 10 shows that our numerical calculations are consistent with these predictions. The intermediate case of $n=1$, which we formally obtain by taking $D a \neq 0$ (specifically $D a=10^{-5}$ and $10^{-2}$ ) and $H=0$, is also shown in this plot. We note that this is a case of more mathematical interest than physical interest since it includes the effects of permeability with a zero thickness substrate. For the case with $D a=10^{-5}$ (and $H=0$ ), the film has not thinned sufficiently in the time interval shown for the dynamics to enter the $n=1$ scaling, and so the dynamics are very similar to the $n=3$ case with $D a=0$. For the case $D a=10^{-2}$, the film thins sufficiently to enter the $n=1$ regime and shows that, while the film thins significantly more than in the $D a=0$ case, there is still no evidence suggesting finite time rupture will occur. This is consistent with the Bertozzi et al. $[45,46]$ theory inasmuch as the $n=1$ case falls into the overlapping range of $n$, where they predict that either finite time or infinite time rupture is possible.

In Figure 11 we present a similar set of calculations that captures the three cases $n=3, n=2$, and $n=0$ within the context of the Beavers-Joseph case with tangentially immobile boundary conditions. In particular, we use $F_{B J T I}$ with $D a=0$ $(n=3), F_{B J T I}$ with $D a \neq 0$ and $H=0(n=2)$, and $F_{B J T I}$ with $D a \neq 0$ and $H \neq 0$ $(n=0)$. Again the $n=3$ case appears consistent with the expected infinite time rupture (in that there is no evidence suggesting finite time rupture) and the $n=0$ case with finite time rupture. The $n=2$ case also appears consistent with infinite time rupture predicted by Bertozzi et al. $[45,46]$, again in the sense that there is no evidence on these time scales indicating finite time rupture.

While the finite time rupture observed in these cases still occurs at long times, particularly in comparison to time scales of interest for contact lens wear, these two sets of calculations provide further documentation that a fundamental change in the thin film rupture dynamics occurs when the underlying porous substrate (contact lens) is present. 
4. Summary and discussion. We have examined a model of a pre-lens tear film on a porous contact lens using lubrication theory to obtain an evolution equation for the dynamics of the tear film. This model is similar to previous ones on tear films in the absence of contact lenses and also to more general fluid dynamics models for fluids on porous layers. We have obtained evolution equations for cases that treat two different slip conditions for two different interfacial conditions on the film surface: stress free and tangentially immobile interface conditions (see Appendix B).

We have examined numerically solutions of our model and have quantified the effects of several parameters such as the Darcy number $D a$, which characterizes the permeability of the contact lens, the contact lens thickness $H$, and also two different slip models: the Beavers-Joseph slip model and the Le Bars-Worster slip model. We have also examined the influence of gravity in this tear film and contact lens model.

For realistic values of $D a$ for contact lenses and for typical time scales between blinks, the influence of the contact lens on the pre-lens film dynamics is small. Despite this, one can still identify the trends in terms of these new processes. We have shown that increasing $D a, H$, or the slip length/depth in either slip model has the overall effect (albeit a small one for realistic parameter values) of increasing the rate of thinning and therefore the likelihood of film rupture and drying of the contact lens. From a more theoretical standpoint, the presence of the underlying porous material introduces new terms in the evolution equation that are significant in that they suggest film rupture occurs in finite rather than infinite time. In particular, these observations, which are consistent with the theory and computations of Bertozzi et al. [45] and Bertozzi [46] show that while the thin film evolution over an impermeable boundary ruptures in infinite time, the introduction of a permeable substrate of nonzero thickness leads to tear film rupture in finite time. Finally, we have observed that the effects of gravity are similar to those observed by Braun and Fitt [3] in the absence of a contact lens. In this case, $D a$ and $H$ again promote film thinning.

We expect that the model developed here may provide a basis upon which more elaborate models of tear films and contact lenses may be investigated. In particular, models that incorporate evaporation and/or the presence of a post-lens film would allow for a quantitative comparison to experimental data and are exciting avenues for future work.

Appendix A. Evolution equation with the Le Bars-Worster slip model. Here we give details for the lubrication theory analysis when the Le Bars-Worster [41] model is used at the liquid-porous boundary. In particular, this model replaces conditions (6)-(8) evaluated at $y=0$ with

$$
\begin{aligned}
& v(x, y=-\delta, t)=V(x, y=-\delta, t), \\
& u(x, y=-\delta, t)=U(x, y=-\delta, t), \\
& p(x, y=-\delta, t)=p(x, y=-\delta, t) .
\end{aligned}
$$

The lubrication analysis proceeds as described in the main text. In the porous region, the fluid velocities are given by (49) and (50), and the pressure is given by (51). In the liquid region, the pressure is also given by (51), but the velocity components take the modified form

$$
u(x, y, t)=\left(-\frac{1}{C a} \frac{\partial^{3} h}{\partial x^{3}}+\epsilon G \cos \theta \frac{\partial h}{\partial x}-G \sin \theta\right)\left[\frac{1}{2}(y+\delta)^{2}-(y+\delta)(h+\delta)-D a\right],
$$


$(\mathrm{A} 5)$

$$
\begin{aligned}
v(x, y, t)= & \left(\frac{1}{C a} \frac{\partial^{4} h}{\partial x^{4}}-\epsilon G \cos \theta \frac{\partial^{2} h}{\partial x^{2}}\right)\left[\frac{1}{6}(y+\delta)^{3}-\frac{1}{2}(h+\delta)(y+\delta)^{2}-D a(y+H)\right] \\
& +\left(-\frac{1}{C a} \frac{\partial^{3} h}{\partial x^{3}}+\epsilon G \cos \theta \frac{\partial h}{\partial x}-G \sin \theta\right)\left[\frac{1}{2}(y+\delta)^{2}\right] \frac{\partial h}{\partial x} .
\end{aligned}
$$

The resulting evolution equation for $h(x, t)$ is given by (52), where $F$ has the form

$$
F_{L W}=\frac{1}{3}(h+\delta)^{3}+D a(h+H)
$$

Appendix B. Evolution equations with the tangentially immobile interface condition. In this section we outline the results of lubrication theory applied to the problem described in the main text with the tangentially immobile boundary condition $\boldsymbol{u} \cdot \hat{\mathbf{t}}=0$ in place of the boundary condition (11). For completeness we present results for both the Beavers--Joseph slip condition as well as the Le Bars-Worster slip condition.

Beavers-Joseph slip condition: In the porous region, the fluid velocities are given by (49) and (50), and the pressure is given by (51). In the liquid region the pressure is also given by (51), but the velocity components take the modified form

$$
u(x, y, t)=\left(-\frac{1}{C a} \frac{\partial^{3} h}{\partial x^{3}}+\epsilon G \cos \theta \frac{\partial h}{\partial x}-G \sin \theta\right)\left[\frac{1}{2}\left(y^{2}-h^{2}\right)+Q_{B J}(y-h)\right],
$$

$$
\begin{aligned}
v(x, y, t)= & \left(\frac{1}{C a} \frac{\partial^{4} h}{\partial x^{4}}-\epsilon G \cos \theta \frac{\partial^{2} h}{\partial x^{2}}\right)\left[\frac{1}{6} y^{3}-\frac{1}{2} h^{2} y+Q_{B J}\left(\frac{1}{2} y^{2}-h y\right)-D a H\right] \\
& +\left(-\frac{1}{C a} \frac{\partial^{3} h}{\partial x^{3}}+\epsilon G \cos \theta \frac{\partial h}{\partial x}-G \sin \theta\right) \\
& \times\left[-h \frac{\partial h}{\partial x} y+\frac{\partial Q_{B J}}{\partial x}\left(\frac{1}{2} y^{2}-h y\right)-Q_{B J} \frac{\partial h}{\partial x} y\right],
\end{aligned}
$$

where

$$
Q_{B J}=\frac{D a-\frac{1}{2} h^{2}}{h+\sqrt{\frac{D a}{\alpha}}} .
$$

The resulting evolution equation for $h(x, t)$ is given by (52), where $F$ has the form

$$
F_{B J T I}=\frac{1}{3} h^{3}+\frac{1}{2} h^{2} Q_{B J}+D a H .
$$

Note that if $D a=0$, one obtains $F_{B J T I}=\frac{1}{12} h^{3}$. If one examines this expression in the limit $D a \ll 1$ and also $H \gg 1$ (so that terms like $h$ are negligible relative to $H$ ), the result is

$$
F_{B J T I} \approx \frac{1}{12} h^{3}+\frac{1}{4} \frac{\sqrt{D a}}{\alpha} h^{2}+D a H+\ldots
$$

Copyright (C) by SIAM. Unauthorized reproduction of this article is prohibited. 
which is $1 / 4$ times $F_{B J}$ under the same approximations if one amplifies the value of $H$ here by a factor of 4 .

Le Bars-Worster slip condition: In the porous region, the fluid velocities are given by (49) and (50), and the pressure is given by (51). In the liquid region the pressure is also given by (51), but the velocity components take the modified form

$$
u(x, y, t)=\left(-\frac{1}{C a} \frac{\partial^{3} h}{\partial x^{3}}+\epsilon G \cos \theta \frac{\partial h}{\partial x}-G \sin \theta\right)\left[\frac{1}{2}\left(y^{2}-h^{2}\right)+Q_{L W}(y-h)\right],
$$

$$
\begin{aligned}
v(x, y, t)= & \left(\frac{1}{C a} \frac{\partial^{4} h}{\partial x^{4}}-\epsilon G \cos \theta \frac{\partial^{2} h}{\partial x^{2}}\right)\left[\frac{1}{6}\left(y^{2}-y \delta+\delta^{2}-3 h^{2}\right)(y+\delta)\right. \\
& \left.+\frac{1}{2} Q_{L W}(y-\delta-2 h)(y+\delta)-D a(-\delta+H)\right] \\
& +\left(-\frac{1}{C a} \frac{\partial^{3} h}{\partial x^{3}}+\epsilon G \cos \theta \frac{\partial h}{\partial x}-G \sin \theta\right) \\
& \times\left[\left(-h \frac{\partial h}{\partial x}+\frac{1}{2} \frac{\partial Q_{L W}}{\partial x}(y-\delta-2 h)-Q_{L W} \frac{\partial h}{\partial x}\right)(y+\delta)\right],
\end{aligned}
$$

where

$$
Q_{L W}=\frac{D a+\frac{1}{2}\left(\delta^{2}-h^{2}\right)}{(\delta+h)} .
$$

The resulting evolution equation for $h(x, t)$ is given by (52), where $F$ has the form

$$
F_{L W T I}=\frac{1}{12}(h+\delta)^{3}-\frac{1}{6} \delta^{3}+D a\left[H+\frac{1}{2}(h-\delta)\right] .
$$

Again note that if $D a=0$ and $\delta=0$, one obtains $F_{L W T I}=\frac{1}{12} h^{3}$. If one examines this expression with $\delta \ll 1$ and $H \gg 1$, the result is

$$
F_{L W T I} \approx \frac{1}{12}(h+\delta)^{3}+D a H+O\left(\delta^{3}, h D a, \delta D a\right),
$$

where, for ease of comparison to $F_{L W}$, we have not expanded the term $(h+\delta)^{3}$ even though it contains higher-order terms in $\delta$. Again we observe that this result is $1 / 4$ times $F_{L W}$ under the same approximations if one amplifies the value of $H$ here by a factor of 4 .

Appendix C. Linear stability of a planar interface. Here we examine the linear stability of a static fluid layer of uniform thickness $h_{0}$. We introduce perturbations

$$
h=h_{0}+h^{\prime}
$$

into (52) and linearize to obtain

$$
h_{t}^{\prime}=-\frac{F\left(h_{0}\right)}{C a} h_{x x x x}^{\prime}+\left[F\left(h_{0}\right) \epsilon G \cos \theta\right] h_{x x}^{\prime}-\left[F^{\prime}\left(h_{0}\right) G \sin \theta\right] h_{x}^{\prime} .
$$

Copyright $\odot$ by SIAM. Unauthorized reproduction of this article is prohibited. 
Now if we seek a perturbation of the form

$$
h^{\prime} \sim e^{\sigma t} e^{i a x}+\text { c.c. }
$$

with growth rate $\sigma$ and wavenumber $a$, we find the characteristic equation

$$
\sigma=-C_{1} a^{4}-C_{2} a^{2}-i a C_{3}
$$

where

$$
C_{1}=\frac{F\left(h_{0}\right)}{C a}, \quad C_{2}=\epsilon F\left(h_{0}\right) G \cos \theta, \quad C_{3}=F^{\prime}\left(h_{0}\right) G \sin \theta .
$$

Note that $C_{1}>0$ always and that $C_{2}, C_{3} \geq 0$ for $0 \leq \theta \leq \pi / 2$. These results show that the effects included in the present model do not destabilize a planar interface since $\operatorname{Real}(\sigma) \leq 0$. The complex growth rate indicates the possibility of wave-like motion with right travelling waves (in the positive $x$ direction) when $\sin \theta>0$ (plane tilted down toward the right) and left travelling waves (in the negative $x$ direction) when $\sin \theta<0$ (plane tilted down toward the left). As noted above, these wave motions will be damped. Note that the different functions $F$, for different slip models and interfacial conditions, influence the decay rate of the perturbations and, in the case of $\sin \theta \neq 0$, the wave speed, but the parameters $D a, \alpha$, and $\delta$ representing permeability and slip are not themselves a source of instability for a planar interface.

Acknowledgments. We would like to thank Richard Braun, Alfa Heryudono, and Stephen Davis for helpful discussions.

\section{REFERENCES}

[1] A. Sharma, R. Khanna, and G. Reiter, A thin film analog of the corneal mucus layer of the tear film: An enigmatic long range non-classical DLVO interaction in the breakup of thin polymer films, Coll. Surf B: Biointerfaces, 14 (1999), pp. 223-235.

[2] Y. L. Zhang, O. K. Matar, and R. V. Craster, Analysis of tear film rupture: Effect of non-Newtonian rheology, J. Coll. Interface Sci., 262 (2003), pp. 130-148.

[3] R. J. Braun And A. D. FitT, Modeling the drainage of the precorneal tear film after a blink, Math. Med. Biol., 20 (2003), pp. 1-28.

[4] I. K. Gipson, Distribution of mucins at the ocular surface, Exp. Eye Res., 78 (2004), pp. 379388.

[5] A. J. Bron, J. M. Tiffany, S. M. Gouveia, N. Yokoi, and L. W. Voon, Functional aspects of the tear film lipid layer, Exp. Eye Res., 78 (2004), pp. 347-360.

[6] I. CHER, Another way to think of tears: Blood, sweat, and ... "Dacruon," The Ocular Surface, 5 (2007), pp. 251-254.

[7] M. A. Lemp, C. Baudouin, J. Baum, M. Dogru, G. N. Foulks, S. Kinoshita, P. Laibson, J. McCulley, J. Murube, S. Pflugfelder, M. Rolando, and I. Toda, The definition and classification of dry eye disease: Report of the Definition and Classification Subcommittee of the International Dry Eye WorkShop (2007), The Ocular Surface, 5 (2007), pp. 75-92; also available online from http://www.theocularsurface.com.

[8] A. Sharma And E. Ruckenstein, Mechanism of tear film rupture and formation of dry spots on cornea, J. Coll. Interface Sci., 106 (1985), pp. 12-27.

[9] H. Wong, I. Fatt, And C. J. Radke, Deposition and thinning of the human tear film, J. Coll. Interface Sci., 184 (1996), pp. 44-51.

[10] K. N. Winter, D. M. Anderson, and R. J. Braun, A model for wetting and evaporation of a post-blink precorneal tear film, Math. Med. Biol., doi:10.1093/imammb/dqp019 (2009).

[11] A. Heryudono, R. J. Braun, T. A. Driscoll, L. P. Cook, and P. E. King-Smith, Singleequation models for the tear film in a blink cycle: Realistic lid motion, Math. Med. Biol., 24 (2007), pp. 347-377.

[12] R. J. Braun and P. E. King-Smith, Model problems for the tear film in a blink cycle: Single equation models, J. Fluid Mech., 586 (2007), pp. 465-490.

Copyright $@$ ( ) by SIAM. Unauthorized reproduction of this article is prohibited. 
[13] K. L. Maki, R. J. Braun, T. A. Driscoll, and P. E. King-Smith, An overset grid method for the study of reflex tearing, Math. Med. Biol.-A, 25 (2008), pp. 187-214.

[14] K. L. Maki, R. J. Braun, W. D. Henshaw, and P. E. King-Smith, Tear film dynamics on an eye-shaped domain I: Pressure boundary conditions, Math. Med. Biol., doi:10. 1093/imammb/dqp023 (2010).

[15] K. L. Maki, R. J. Braun, P. Ucciferro, W. D. Henshaw, and P. E. King-Smith, Tear film dynamics on an eye-shaped domain II: Flux boundary conditions, J. Fluid Mech., 647 (2010), pp. 361-390.

[16] J. J. Nichols And P. E. King-Smith, Thickness of the pre- and post-contact lens tear film measured in vivo by interferometry, Invest. Ophthalmol. Visual Sci., 44 (2003), pp. 68-77.

[17] J. J. Nichols And P. E. King-Smith, The impact of hydrogel lens settling on the thickness of the tears and contact lens, Invest. Ophthalmol. Visual Sci., 45 (2004), pp. 2549-2554.

[18] J. J. Nichols, G. L. Mitchell, And P. E. King-Smith, Thinning rate of the precorneal and prelens tear films, Invest. Ophthalmol. Visual Sci., 46 (2005), pp. 2353-2361.

[19] R. M. Pearson, Karl Otto Himmler, manufacturer of the first contact lens, Contact Lens Ant. Eye, 30 (2007), pp. 11-16.

[20] C. Maldonado-Codina And N. Efron, Impact of manufacturing technology and material composition on the clinical performance of hydrogel lenses, Optometry Vision Sci., 81 (2004), pp. 442-454.

[21] D. F. SweEney, The Max Schapero Memorial Award Lecture 2004: Contact lenses on and in the cornea, what the eye needs, Optometry Vision Sci., 83 (2006), pp. 133-142.

[22] V. Guryča, R. Hobzová, M. PřÁdnÝ, J. Širc, And J. MicháleK, Surface morphology of contact lenses probed with microscopy techniques, Contact Lens Ant. Eye, 30 (2007), pp. 215222.

[23] P. E. RAAd And A. S. SABau, Dynamics of a gas permeable contact lens during blinking, Trans. ASME, J. Appl. Mech., 63 (1996), pp. 411-418.

[24] M. V. Monticelli, A. Chauhan, and C. J. Radke, The effect of water hydraulic permeability on the settling of a soft contact lens on the eye, Curr. Eye. Res., 30 (2005), pp. 329-336.

[25] A. Chauhan and C. J. Radke, Settling and deformation of a thin elastic shell on a thin fluid layer lying on a solid surface, J. Coll. Interface Sci., 245 (2002), pp. 187-197.

[26] F. Fornasiero, J. M. Prausnitz, and C. J. Radke, Post-lens tear-film depletion due to evaporative dehydration of a soft contact lens, J. Membrane Sci., 275 (2006), pp. 229-243.

[27] L. C. Thai, A. Tomlinson, and M. G. Doane, Effect of contact lens material on tear physiology, Optometry Vision Sci., 81 (2004), pp. 194-204.

[28] W. Mathers, Evaporation from the ocular surface, Exp. Eye Res., 78 (2004), pp. 389-394.

[29] C. W. McMonnies, Incomplete blinking: Exposure keratopathy, lid wiper epitheliopathy, dry eye, refractive surgery, and dry contact lenses, Contact Lens Ant. Eye, 30 (2007), pp. 3751.

[30] R. L. Chalmers And C. G. Begley, Dryness symptoms among an unselected clinical population with and without contact lens wear, Contact Lens Ant. Eye, 29 (2006), pp. 25-30.

[31] S. H. Davis AND L. M. Hocking, Spreading and imbibition of viscous liquid on a porous base, Phys. Fluids, 11 (1999), pp. 48-57.

[32] S. H. DAvis AND L. M. HockING, Spreading and imbibition of viscous liquid on a porous base. II, Phys. Fluids, 12 (2000), pp. 1646-1655.

[33] O. Devauchelle, C. Josser, and S. Zaleski, Forced dewetting on porous media, J. Fluid Mech., 574 (2007), pp. 343-364.

[34] J. M. Acton, H. E. Huppert, And M. G. Worster, Two-dimensional viscous gravity currents flowing over a deep porous medium, J. Fluid Mech., 440 (2001), pp. 359-380.

[35] J. P. PAScal, Linear stability of fluid flow down a porous inclined plane, J. Phys. D: Appl. Phys., 32 (1999), pp. 417-422.

[36] I. M. R. SAdiq And R. Usha, Thin Newtonian film flow down a porous inclined plane: Stability analysis, Phys. Fluids, 20 (2008), 022105.

[37] G. S. Beavers and D. D. JosePh, Boundary conditions at a naturally permeable wall, J. Fluid Mech., 30 (1967), pp. 197-207.

[38] G. I. TAYLOR, A model for the boundary condition of a porous material. Part 1, J. Fluid Mech., 49 (1971), pp. 319-326.

[39] S. Richardson, A model for the boundary condition of a porous material. Part 2, J. Fluid Mech., 49 (1971), pp. 327-336.

[40] G. Neale and W. Nader, Practical significance of Brinkman's extension of Darcy's law: Coupled parallel flows within a channel and a bounding porous medium, Can. J. Chem. Eng., 52 (1974), pp. 475-478.

Copyright $@$ by SIAM. Unauthorized reproduction of this article is prohibited. 
[41] M. Le Bars And M. G. Worster, Interfacial conditions between a pure fluid and a porous medium: Implications for binary alloy solidification, J. Fluid Mech., 550 (2006), pp. 149173.

[42] P. E. King-Smith, B. A. Fink, J. J. Nichols, K. K. Nichols, R. J. Braun, and G. B. MCFADDEN, The contribution of lipid layer movement to tear film thinning and breakup, Invest. Ophthalmol. Visual Sci., 50 (2009), pp. 2747-2756.

[43] H. P. Greenspan, On the motion of a small viscous droplet that wets a surface, J. Fluid Mech., 84 (1978), pp. 125-143.

[44] L. M. HockIng, Sliding and spreading of thin two-dimensional drops, Quart. J. Mech. Appl. Math., 34 (1981), pp. 37-55.

[45] A. L. Bertozzi, M. P. Brenner, T. F. Dupont, and L. P. Kadanoff, Singularities and similarities in interface flows, in Trends and Perspectives in Applied Mathematics, Appl. Math. Sci. 100, L. Sirovich, ed., Springer-Verlag, New York, 1994, pp. 155-208.

[46] A. L. BertozzI, Symmetric singularity formation in lubrication-type equations for interface motion, SIAM J. Appl. Math, 56 (1996), pp. 681-714.

[47] N. G. Hadjiconstantinou, Comment on Cercignani's second-order slip coefficient, Phys. Fluids, 15 (2003), pp. 2352-2354.

[48] J. Mauer, P. Tabeling, P. Joseph, and H. Willaime, Second-order slip laws in microchannels for helium and nitrogen, Phys. Fluids, 15 (2003), pp. 2613-2621.

[49] M. Beerman AND L. N. Brush, Oscillatory instability and rupture in a thin melt film on its crystal subject to freezing and melting, J. Fluid Mech., 586 (2007), pp. 423-448.

[50] R. J. BRAUN, private communication, 2007.

Copyright (c) by SIAM. Unauthorized reproduction of this article is prohibited. 